\title{
Erratum to: Algebra and logic for access control
}

\author{
Matthew Collinson and David Pym \\ Hewlett-Packard Laboratories, Long Down Avenue, Stoke Gifford, Bristol BS34 8QZ, UK \\ E-mail: collision@hp.com; david.pym@hp.com
}

\section{Erratum to: Form Asp Comp (2010) 22: 83-104 DOI 10.1007/s00165-009-0107-x}

A particular type of error was made in certain proofs regarding the relation $\approx$. The errors regarding this and their limited consequences are listed below.

1. Replace the words 'a congruence' by 'an equivalence' in Proposition 4.1 and the sentence immediately preceeding it.

2. In Proposition 4.1 delete the second enumerated point and the sentence immediately following the proof.

3. Replace the proof of Proposition 4.1 by the following sentence: 'The proofs of all parts of this follow from the definition of $\approx$ and by applying the standard methods for bisimulation relations.'

4. Delete the third, fifth, sixth, seventh and eighth enumerated points from Proposition 4.2 and replace the proof of 4.2 with: 'Again, the proofs of these points are direct uses of the definition of simulation.'

5. The sentence immediately following the proof of Proposition 4.2 should be replaced by: 'The above results tell us that we have a system that formally reconstructs the following natural properties of roles: any agent acting in one of its roles is less powerful (has fewer or equal capabilities) than the original agent; an agent $E$ in the role $E$ is as powerful as the agent $E$; if $E$ is less powerful than $F$ then every role of $E$ is less powerful than the corresponding role of $F$.'

6. Delete the fifth points from both Proposition 5.1 and its proof.

7. In Sect. 8 (lines 9,10 ) replace $\approx$ by $\sim$ in the unit property for $\times$ and the associativity property for $\propto$.

These do not affect the main results of Theorems 5.1 and 5.2. They do not affect the access control examples of Sect. 6. The only points to be retracted in Sect. 7 (lines 13,14) are the claims that a version of the sixth point of Proposition 4.2, and consequently the fifth point of Proposition 5.1, hold in the set-up using $\sim$. These two points are recovered in the presence of the frame rule discussed in Sect. 8. All of the other claims in Sect. 7 remain correct as originally stated.

References to the fact that $\approx$ is a congruence contained in the earlier papers [CPT07, PT06, PT07] are also incorrect: each such statement should be replaced by a statement that $\approx$ is an equivalence.

\section{References}

[CPT07] Collinson M, Pym D, Tofts C (2007) Errata for Formal Aspects of Computing (2006) 18:495-517 and their consequences. Form Asp Comput 19(4):551-554

The online version of the original article can be found under doi:10.1007/s00165-009-0107-x.

Correspondence and offprint requests to: M. Collinson and D. Pym, E-mail: collision@hp.com; david.pym@hp.com 
Pym D, Tofts C (2006) A calculus and logic of resources and processes. Form Asp Comp 18(4):495-517 (Errata in [CPT07]) Pym D, Tofts C (2007) Systems modelling via resources and processes: philosophy, calculus, semantics, and logic. In: Cardelli L, Fiore M, Winskel G (eds) Computation, meaning and logic: articles dedicated to Gordon Plotkin. Electronic notes in theoretical computer science, vol 107. Elsevier, Amsterdam, pp 545-587 (Errata in [CPT07])

Communicated 15 June 2009 by J.V. Tucker

Published online 21 April 2010 